\title{
Académicos gestores en la universidad actual: Desafíos y aprendizajes
}

\author{
Marita Sánchez-Moreno(iD, Mariana Altopiedi (iD \\ Universidad de Sevilla (Spain) \\ marita@us.es, maltopiedi@us.es
}

Received November, 2015

Accepted January, 2016

\section{Resumen}

Objeto: El artículo presenta y discute algunos resultados de un estudio acerca de la gestión de académicos universitarios y se centra en la identificación de las principales claves -tanto de contenido como de orden metodológico- para diseñar programas formativos en relación con esta tarea.

Diseño/metodología/enfoque: La investigación -de corte cualitativo- contempló la realización de entrevistas en profundidad a 71 gestores académicos universitarios positivamente valorados como tales, en tres universidades españolas -Universidad de Las Palmas de Gran Canaria (ULP), Universidad de Lleida (UdL) y Universidad de Sevilla (US). Los datos fueron analizados mediante el programa MAXQDA.10 y la interpretación de los mismos fue contrastada durante una segunda ronda de entrevistas a 15 de estos académicos gestores.

Aportaciones y resultados: Los resultados sugieren la existencia de un cierto "ciclo vital" en los académicos gestores y nos informan de sus razones más o menos conscientes para continuar en el ejercicio de los cargos, de sus preocupaciones en torno del manejo de las relaciones interpersonales y la gestión de conflictos, así como de la necesidad percibida de recibir una formación para el desarrollo de la labor de gestión basada en una metodología activa y participativa, favorecedora de un aprendizaje práctico. 
Limitaciones: Las principales limitaciones del estudio derivan de dos cuestiones. La primera, de orden metodológico, está relacionada con el tamaño relativamente pequeño de la muestra. La segunda, por su parte, se refiere al momento de transición en el modelo de gobierno y gestión de las universidades que puede suponer un cambio en el modo en que los académicos se preparen para llevar a cabo estas funciones así como en su valoración e interés por desarrollarlas.

Implicaciones prácticas: Dado que era esa una de las finalidades del estudio del que surge la información presentada aquí, los resultados del mismo han permitido el diseño de un Programa formativo para la gestión universitaria, cuya primera edición ya ha tenido lugar.

Implicaciones sociales: La puesta en marcha de Programas de Formación en Gestión Académica Universitaria como el derivado del estudio presentado puede contribuir a la mejora del funcionamiento de las instituciones universitarias.

Originalidad / Valor añadido: Dada la escasez de propuestas formativas como la surgida de los hallazgos aquí presentados, la importancia de los mismos se ve reforzada.

Palabras clave: Liderazgo universitario, Gestión académica, Formación en gestión académica, Desarrollo profesional, Buenas prácticas en gestión

Códigos JEL: I2, I23

Title: Academic managers in contemporary university: Challenges and learnings

\section{Abstract}

Purpose: The article presents and discusses some findings derived from a research project about University academic management, and it is focused on the identification of principal clues -even related to subjects and methodology- for the design of training programmes on this task.

Design/methodology: The research design adopted a qualitative approach and contemplated the development of in-depth interviews to 71 academic managers at the university who were valued as good in that task, in three Spanish Universities -Universidad de Las Palmas de Gran Canaria (ULP), Universidad de Lleida (UdL) y Universidad de Sevilla (US). Data were analyzed using MAXQDA.10 and their interpretations were contrasted with the opinions obtained in a second round of interviews with 15 of those managers. 
Findings: Results allows to identify some kind of "vital cycle" in academic managers, inform about their reasons to continue in their managing charges, about their worries related to interpersonal relationships and conflict management, as well as about the recognized need of receiving a specific training for academic management, based on an active a participative methodology which can promote a practical learning.

Research limitations/implications: The most important limitations of the study have to do with two unrelated issues. First of them, from a methodological point of view, is related to the limited number of participants. Second of them derives of the changing environment in which management and government model of Spanish is changing. This could probably affect the value and the interest showed by an academics on University management.

Practical implications: According to one of the original mains of the study, a training program for academics in university management was already designed and developed.

Social implications: The Development of Training Programs on University Management as the one derived from the commented study would represent a contribution for the improvement of Universities' functioning.

Originality/value: Taking into account the limited number of training programs based on empirical evidenced training needs, the relevance of the proposals derived from this study is reinforced.

Keywords: Leadership at the University, Academic management, Training on academic management, Professional development, Management good practices

Jel Codes: I2, I23

\section{Introducción}

\subsection{La universidad actual y sus desafíos para la gestión}

En las tres últimas décadas cabe reconocer múltiples transformaciones en la Universidad, entre las que destaca la rápida expansión tanto del número de estudiantes como del de centros universitarios que, como señala Goodwill (2012) citando a Sharrock (2007), ha forzado importantes modificaciones en las organizaciones de este nivel en las sociedades desarrolladas. Asimismo, deben contemplarse particularidades del sistema español, que "tras la promulgación de la Ley de Reforma Universitaria 
(1983), ha experimentado una transformación substancial y positiva que ha dado como resultado un sistema muy distinto del que la democracia heredó del franquismo" (Corominas Subias \& Sacristán Adinolfi, 2011: pp. 58-59). Absorber este crecimiento ha estado a cargo casi exclusivamente de la universidad pública.

Simultáneamente a la democratización del acceso al conocimiento y las acreditaciones, se exige a la Universidad que no abandone su tradicional función en la selección y formación de élites, percibiéndose un incremento y diversificación de las expectativas y demandas.

Otro factor de complejización interna es el creciente peso de la investigación entre las misiones de la Universidad, que ha repercutido en la identidad de los académicos al punto que -como señalan Jiménez-Contreras, De Moya Anegón y Delgado López-Cózar (2003)- ni aún la marcada retracción de la inversión en educación y ciencia producida por la crisis económica de los inicios de los '90 produjo el descenso de la producción científica en los niveles previsibles.

En este contexto, muchas voces reclaman un aumento de la participación privada en la universidad y la opción por políticas de financiamiento basadas en la competitividad, proponiendo como modelo algunas universidades estadounidenses. La implantación de estas políticas -que implica un acercamiento del campo educativo al económico- es apreciable en el conjunto del ámbito universitario europeo como evidencia el análisis de Deer (2003) sobre las universidades británica y francesa- y se ve respaldado por argumentos liberales como el de la democratización y la autonomía.

Paralelamente, se ha evidenciado una conmoción de la lógica de la organización universitaria, que fue pasando del tradicional modelo vertical basado en la cátedra y en su figura central, a un modelo matricial, en que la unidad organizativa fundamental es el Departamento (Solé Parellada \& Llinàs-Audet, 2011: pp. 20). Propia de este modelo -afianzado en la universidad española a partir de los años '80- es la diversificación de los servicios, con la consiguiente complejización de los procesos de gestión y administración. En consecuencia, el propio sistema de gobierno universitario parece verse puesto en cuestión, como sugieren Corominas Subias y Sacristán Adinolfi (2011: pp. 76) al atribuir al sistema actual cierta responsabilidad en la aparición de algunos problemas de la universidad española, como las deficiencias en la planificación global, la presencia excesiva de intereses corporativos en las decisiones y cierta laxitud en la gestión presupuestaria. Para afrontar estos desafíos se propone un replanteamiento del modelo organizativo como universidad moderna (Solé Parellada \& Llinàs-Audet, 2011), un modelo de planificación estratégica que exige líderes que la lleven adelante y sitúa la gestión en la línea de la gobernabilidad" (Murillo García \& Salas Durazo, 2011). 
Este aumento de la complejidad organizativa supone tres efectos interrelacionados sobre la gestión y quienes la ejercen:

- La sobrecarga y diversificación de tareas, que exige nuevas capacidades a los líderes institucionales.

- El incremento en la amplitud y diversidad de los conocimientos requeridos.

- La eventual necesidad de instancias formativas ajustadas a la diversidad de los conocimientos y competencias requeridos por la gestión, así como a sus destinatarios.

Relacionaremos a continuación estas cuestiones con el conocimiento existente:

- En el marco de estas transformaciones y las exigencias de adaptación a las presiones externas sufridas por la Universidad en las últimas décadas, la gestión y el liderazgo de estas organizaciones se han visto sujetos a una incertidumbre creciente. Esto, sumado a la difusión de discursos como el del "nuevo gerencialismo" (Deem, 1998, 2005) condujo a superar la visión del gestor universitario como mero agente administrativo y como individuo que actúa aisladamente. Como desarrollamos en otro trabajo (Sánchez-Moreno \& López-Yañez, 2013), algunos indicadores de buenas prácticas en la gestión universitaria son: liderazgo compartido o distribuido, los procesos colegiados de toma de decisiones y el equilibrio entre lo pedagógico y lo organizativo. Se reconocen aquí capacidades de los gestores universitarios entre las que destacan tanto las de orden cognitivo como las de tipo personal e interpersonal (Scott, Coates \& Anderson, 2008), relativas a la capacidad para motivar y dirigir grupos así como para controlar las emociones y el comportamiento propios.

- A pesar del aparente acuerdo sobre las habilidades y competencias oportunas para el ejercicio de la gestión universitaria, conviene contemplar la incidencia de factores contextuales y personales, tales como el área de conocimiento y el momento vital.

En relación con el primero de los aspectos señalados, estudios como el de Nguyen (2012) subrayan la conveniencia de, más allá de "capacidades genéricas" para la gestión, atender a las demandas de cada organización, así como a los conocimientos con que cuentan los gestores. Con respecto a la biografía personal, Castro $(2010,2011)$ señala que el ejercicio de la gestión puede ser una instancia formativa, por lo que la prioridad con que se requiere una u otra capacidad va variando a lo largo del desarrollo profesional. Diferencia, aquí, tres fases: una inicial, caracterizada por la necesidad de familiarizarse con el cargo y comprender las dinámicas institucionales; una segunda etapa, donde la actividad se orienta al desarrollo del proyecto, y una tercera, preocupada por el relevo. 
Otros supuestos condicionantes de los saberes y capacidades de los gestores son el género y el área de conocimiento. Mientras que la importancia del primer factor es cuestionada por nuestros estudios previos (Sánchez-Moreno, 2008, 2009), la del segundo de ellos es relativizada por Del Favero (2006), que subraya el papel de las experiencias - propias o de carácter vicario- en el aprendizaje de la gestión.

Esto último plantea la tercera de las cuestiones en debate, la conveniencia de la formación de los académicos en gestión, atendiendo a las orientaciones derivadas de los escasos pero relevantes estudios a este respecto:

- Orientación hacia la formación de una identidad como directivo universitario (Wolverton, Ackerman \& Holt, 2005; Aasen \& Stensaker, 2007).

- Énfasis en el conocimiento práctico, el intercambio de experiencias y el análisis de situaciones reales (Aasen \& Stensaker, 2007; Calabrese et al., 2008), así como la mentorización y la inclusión de la formación como estrategia que dé continuidad a la gestión (Mc Murray, Hnely, Chaboyer, Clapto, Lizzio \& Teml, 2012).

- Vinculación de la formación con el discurso de la colegialidad y la colaboración, evitando visiones gerencialistas (Aasen \& Stensaker, 2007; Calabrese et al., 2008).

- Respeto por las necesidades y carcacterísticas de las organizaciones (Aasen \& Stensaker, 2007; Calabrese et al., 2008).

- Incorporación de las aplicaciones tecnológicas en la gestión así como para favorecer el intercambio y la colaboración (Calabrese et al., 2008).

- Inclusión de la formación en el marco de una renovación del conjunto de la organización, en sus aspectos estructurales y culturales (Aasen \& Stensaker, 2007).

Además de estas orientaciones específicas conviene contemplar cuestiones identificadas por Pulido, Aguilar y Alemán (2010) en un estudio comparado sobre la formación de directivos universitarios, así como las directrices de la Leadership Foundation for Higher Education en el año 2009: diversidad, internalización, interconexión y desarrollo sostenible. 


\subsection{Método}

El principal objetivo de la investigación fue diseñar, desarrollar y evaluar un programa de formación en gestión específicamente dirigido a académicos universitarios. Para lograrlo, nos propusimos en primera instancia y cuyos resultados son los que se muestran en este artículo, conocer el perfil de los gestores así como prácticas exitosas que ayudaran a visualizar aspectos de la práctica dignos de contemplarse en programas destinados a la formación en estas tareas.

\subsection{Muestra}

Se adoptó una metodología de corte cualitativo, realizando entrevistas en profundidad a gestores académicos de las universidades de Las Palmas de Gran Canaria (ULP), Lleida (UdL) y Sevilla (US), seleccionados atendiendo al tamaño, la trayectoria histórica y la pertenencia de investigadores del estudio.

Los 71 entrevistados fueron elegidos mediante la consulta a informantes clave en cada universidad (18 de ULP, 21 de UdL y 32 de US). La muestra (Tabla 1) se conformó considerando como criterios: género, cargo académico, trayectoria en cargos académicos y área de conocimiento. 


\begin{tabular}{|c|c|c|c|c|c|c|c|c|c|c|c|c|c|c|c|}
\hline & \multicolumn{2}{|c|}{ Sexo } & \multicolumn{5}{|c|}{ Área de conocimiento } & \multicolumn{5}{|c|}{ Cargo } & \multicolumn{3}{|c|}{ Universidad } \\
\hline & $\mathbf{F}$ & $\mathbf{M}$ & Hm. & Cien. & Scl. & Téc. & San & Rectorado & Decanato & Dpto. & S.exp. & Otros & US & $\mathbf{U d L}$ & ULP \\
\hline $\mathbf{F}$ & 32 & & 4 & 2 & 21 & 3 & 2 & $\begin{array}{r}5 \\
\text { (vice) }\end{array}$ & $\begin{array}{r}7 \\
(5 \text { vicedec., } \\
1 \text { decana, } \\
1 \text { ex-dec.) }\end{array}$ & $\begin{array}{r}9 \\
(6 \text { director., } \\
1 \text { ex-dir., } \\
1 \text { secret., } \\
1 \text { jefa } \\
\text { estudios })\end{array}$ & 6 & 4 & 15 & 11 & 6 \\
\hline $\mathbf{M}$ & & 39 & 4 & 5 & 20 & 5 & 5 & $\begin{array}{r}5 \\
(1 \text { vice, } \\
1 \text { adjunto, } \\
2 \text { rectores, } \\
1 \text { ex-rect. })\end{array}$ & $\begin{array}{r}9 \\
(7 \text { decanos, } \\
1 \text { vicedec., } \\
1 \text { secr.) } \\
\end{array}$ & $\begin{array}{r}12 \\
\quad \text { (7dir., } \\
2 \text { ex-dir., } \\
1 \text { ex-secret. } \\
1 \text { jefe est.) }\end{array}$ & 6 & 8 & 17 & 10 & 12 \\
\hline $\mathrm{Hm}$. & 4 & 4 & 8 & & & & & 1 & 1 & 2 & 3 & 1 & 4 & 1 & 3 \\
\hline Cien. & 2 & 5 & & 7 & & & & 3 & 0 & 1 & 2 & 1 & 3 & 1 & 3 \\
\hline Sclc. & 21 & 20 & & & 41 & & & 3 & 11 & 13 & 6 & 8 & 20 & 14 & 7 \\
\hline Téc. & 3 & 5 & & & & 8 & & 2 & 1 & 3 & 0 & 2 & 2 & 2 & 4 \\
\hline San. & 2 & 5 & & & & & 7 & 1 & 3 & 2 & 1 & 0 & 3 & 3 & 1 \\
\hline Rect. & 5 & 5 & 1 & 3 & 3 & 2 & 1 & 10 & & & & & 2 & 4 & 4 \\
\hline Dcto. & 7 & 9 & 1 & 0 & 11 & 1 & 3 & & 16 & & & & 6 & 5 & 5 \\
\hline Dpto. & 9 & 12 & 2 & 1 & 13 & 3 & 2 & & & 21 & & & 11 & 7 & 3 \\
\hline S.exp. & 6 & 6 & 3 & 2 & 6 & 0 & 1 & & & & 12 & & 10 & 1 & 1 \\
\hline Otros & 4 & 8 & 1 & 1 & 8 & 2 & 0 & & & & & 12 & 4 & 4 & 4 \\
\hline US & 15 & 17 & 4 & 3 & 20 & 2 & 3 & 2 & 6 & 10 & 10 & 4 & 32 & & \\
\hline UdL & 11 & 10 & 1 & 1 & 14 & 2 & 3 & 4 & 5 & 7 & 1 & 4 & & 21 & \\
\hline ULP & 6 & 12 & 3 & 3 & 7 & 4 & 1 & 4 & 5 & 4 & 1 & 4 & & & 18 \\
\hline
\end{tabular}

Tabla 1. Muestra 


\subsection{Instrumentos}

Utilizamos la entrevista en profundidad y nos servimos de un guión diseñado ad hoc por el equipo de investigación, que abordaba datos biográficos e información sobre acceso a la gestión y motivaciones para ello. Además, se indagaba en situaciones significativas vividas en la gestión, las repercusiones personales y organizativas, y los aprendizajes alcanzados. Finalmente, se preguntaba sobre la necesidad de formación en gestión, sus contenidos y modalidades.

\subsection{Procedimiento}

Las entrevistas fueron registradas en audio. Los datos se analizaron mediante una codificación temática y con el apoyo del software para datos cualitativos MAXQDA.10, empleando un sistema de categorías fundado en las dimensiones consideradas en el guión. Tras una primera puesta a prueba y posterior ajuste de este instrumento de análisis, se codificó el total de las entrevistas, se procedió a la triangulación de resultados entre investigadores y se formularon las primeras interpretaciones, sometidas a validación mediante una segunda ronda de entrevistas. Los participantes en ella fueron 15 gestores académicos de la muestra, seleccionados atendiendo a su experiencia y su disposición para colaborar en la mejora del ejercicio de la gestión. Estas entrevistas se desarrollaron a partir de un nuevo guión diseñado en relación con las interpretaciones de los hallazgos obtenidos en la primera ronda de entrevistas.

Las situaciones dilemáticas narradas por cada entrevistado se consideraron de manera independiente, se sistematizaron siguiendo un protocolo diseñado ad hoc y se analizó su contenido. Luego, se seleccionaron algunas representativas de diferentes temáticas, para ser desarrolladas bajo pautas comunes hasta adoptar el formato caso. El conjunto de casos diseñados forman parte de los materiales de trabajo del programa formativo diseñado para gestores académicos universitarios. 


\section{Resultados y discusión}

\subsection{Datos biográficos}

Una tendencia observable entre los gestores entrevistados es la continuidad en la gestión, aunque varíen el cargo o el nivel jerárquico. Esto apoya la idea de un cierto "ciclo vital" del gestor universitario (Castro, 2010), desde una etapa inicial de escasa confianza en la propias capacidades gestoras por falta de conocimiento específico, seguida de una etapa de desarrollo en que la adquisición del mismo permite cimentar las propias actuaciones y conlleva desarrollo profesional, hasta una etapa final en que se abandona la tarea directiva. Esta interpretación se corrobora en las entrevistas de contraste:

"Totalmente de acuerdo. Esas son las fases..." [...] Vas perdiendo impetu [...] vas ganando serenidad en los planteamientos y te vas haciendo más flexible... más permeable a las ideas de los otros" (MCA_US 2).

"Como todo ciclo tiene una parte de muerte que es la que hay que hacer más corta, [...] la gente se va manteniendo en el cargo y llega el momento que ya ha innovado, le parece que lo está haciendo bien y alli se estabiliza. Y como es lo cómodo seguir haciendo lo mismo, creo que hay un momento que también habría que acortar el final de las carreras de gestión de las personas." (MM_ULPGC 2)

En relación con el área de conocimiento, cabe observar un predominio en la muestra -del campo socio-jurídico. Una primera interpretación, puede atribuir este sesgo a la pertenencia disciplinar de los informantes, que pudieran estar orientados a reconocer la labor de miembros de sus propias Facultades. Otra posible explicación es sugerida por uno de los participantes, quien al ser entrevistado sostiene la relativa ventaja que representa la formación en Ciencias Sociales a la hora de realizar tareas que implican conocimientos propios de la misma, como el de la normativa, la organización y el manejo de grupos y de personas, la gestión económica, etc. Cabe pensar, por tanto, que se presume que quienes cuentan por su formación profesional con conocimientos cercanos al campo de la gestión tienen mayores posibilidades de desempeñarse eficazmente en ella y, por tanto, se sienten más inclinados a asumirla.

Asimismo, este fenómeno podría estar relacionado con la orientación profesionalizante de muchas de las carreras del área de las Ciencias Sociales. Este hecho parece hacer a estos académicos más propensos a combinar los diferentes roles del profesor universitario -la docencia, la gestión y la investigación. 


\subsection{En relación con el acceso a la gestión}

Un rasgo común al conjunto de la muestra es la ausencia de formación específica para el desempeño de la gestión que contrasta con la actual tendencia a la profesionalización de esta actividad señalada por Maassen y Paussits (2013) pero coincide claramente con la escasez de programas de formación en liderazgo y gestión en el ámbito europeo evidenciada por el estudio de estos autores.

"No be becho nada de formación, es todo de forma autodidacta, he ido aprendiendo con la experiencia y he asistido a algún cursillo pero casi no existen cursos de gestión generales, existen grandes cursos, tipo Máster pero evidentemente la dedicación a la universidad te impide asistir". JR_UdL, 3-4.

“(...) Yo be sido un gestor "amateur", autodidacta.” JMG_US, 3-4.

En cambio, buena parte de los gestores reconoce haber ido aprendiendo durante el desempeño de la tarea, merced al apoyo de personas más experimentadas, mediante la observación de otros gestores o, incluso, a partir de su tutorización más o menos informal, cuya importancia es también reconocida en las entrevistas de contraste.

"Para mi fue importante contar con la figura de aquel decano". CM_US, 11- 11.

“... son cosas que se aprenden al lado de alguien (...) como aprendi, realmente, gestión, fue gestionando. De alguna manera, la formación de los equipos se hace desde dentro. Entras desde un puesto más periférico y abí vas viendo cómo..." (MCA_US 2).

Las dificultades iniciales por ausencia de formación pueden verse como refuerzo del sentimiento de logro y desafío ante los retos, dando lugar a un fenómeno semejante al descrito por la literatura acerca de los liderazgos femeninos en ámbitos tradicionalmente ocupados por hombres: el de la "abeja reina". Conseguir algo reservado a pocas personas - por su condición de mujeres, en aquel caso- funciona como indicador de que la o el "elegido" tiene méritos para serlo.

Otra importante fuente de aprendizaje vicario parece provenir del personal de administración y servicios que con frecuencia aporta soluciones a tareas habituales pero desconocidas para quien se inicia en la dirección. Sin embargo, tal y como señalamos en otro trabajo (Sánchez-Moreno \& López-Yáñez, 2013), existe un problema arquetipo en relación en la gestión en las universidades españolas entre el trabajo de los gestores académicos y los técnicos de la gestión, que hace que las decisiones se tomen en dos niveles: el político y el técnico. Mientras que en el nivel académico la toma de decisiones se consensúa, en los servicios técnicos es jerarquizada. Investigaciones como la de Castro (2011) confirman que una inadecuada relación entre estos dos colectivos puede convertirse en una importante fuente de preocupación o incomodidad. 
Lo antedicho se relaciona con el modo de acceder a la función directiva predominante en nuestra muestra. Mayoritariamente, los entrevistados manifiestan no haber previsto desempeñarse como gestores antes que alguien -frecuentemente, una persona con quien trabajaban en otros campos académicos- les solicitase su colaboración y/o acompañamiento.

"El que accede a decano era P., un compañero, que además somos amigos, y entonces suele pasar: cuando uno tiene un cargo de estos se rodea de gente con la cual bay buen entendimiento, y entonces me pidió que ya que él iba de decano, porque yo le animé a que fuera de decano, el me dijo: pues tú te vienes a jefe de estudios." (RA_UdL, $5-5)$.

Mientras que el primer cargo de gestión se asume por designación, integrándose en un equipo formado por alguien que asume su cargo por elección, una vez demostrada la capacidad gestora, se opta posteriormente a cargos electivos.

Parecería que el ejercicio del primer cargo funciona como entrenamiento o formación en la práctica que, una vez concluido con éxito, asegura la capacidad para asumir nuevos desafíos de gestión. Sería tanto un espacio de formación como una prueba de la capacidad directiva, ante el entorno y ante sí mismo. Supondría la etapa inicial en un ciclo vital que tiende a tener continuidad, toda vez que el gestor comprueba su valía, adquiere conocimientos que le dan soltura en el ejercicio de la tarea y ésta pasa a ser no sólo un desafío sino una fuente de satisfacción. Esta descripción sobre el acceso a la gestión es confirmada por una de las participantes en las entrevistas de contraste:

“... mucho de 'estar en el momento' al lado de la persona que te abre la puerta... aunque no seas la mejor pero estabas ahi” (MO_US 2).

Interpretaciones formuladas por López-Yáñez y Sánchez-Moreno (2008) sostenían que muchas de las directivas participantes en el estudio habían accedido a la gestión motivadas por el requerimiento de quien fuera su mentor - un hombre, generalmente- y asumir esa tarea había sido para ellas una forma de mostrar gratitud a esta persona.

Otras coincidencias de nuestros hallazgos con los del estudio anterior se refieren a la llegada a la tarea directiva mediante la aceptación de un cargo, por designación de una persona allegada. En segundo término, a que esta experiencia de gestión es seguida por otras, en muchos casos derivadas de la elección. Finalmente, destaca la implicación en un proyecto colectivo como motivo de esta continuidad. En este sentido, los entrevistados encuentran que su compromiso con la organización o el grupo son las motivaciones principales para acceder al cargo. El sentimiento de obligación a cumplir se señala como causa, tanto de la aceptación inicial como de la continuidad en la labor gestora. Tanto es así que 
sólo una de las personas entrevistadas inicialmente reconoce que le gusta dirigir y disfruta ejerciendo cargos de gestión. El resto tiende a entenderla como una obligación ligada al cumplimiento de un plan cuya consecución considera una responsabilidad personal. Abandonar la tarea sin alcanzarlo parece suponer una forma de deslealtad o un riesgo para el éxito alcanzado gracias al esfuerzo previo.

"La motivación para acceder al cargo ha sido en ambos casos las responsabilidad, no me gustan los cargos, no me gustan en absoluto y bueno la primera vez sentí que ya me tocaba... [...] Senti que me tocaba, que era una cuestión de responsabilidad. En nuestra facultad y sé que en otras también cada vez es más difícil encontrar a alguien que quiera hacerse cargo de este tipo de cargos y teniamos problemas porque babia gente que no quería". AA_ULP, 2-2.

Aunque esto es casi un rasgo de la cultura occidental de raíz judeocristina, parecería que el placer o el interés personal son vividos con culpa por quienes desempeñan labores directivas en la Universidad.

"Parece que está mal visto que te guste gestionar personas, como si fuera un toque de vanidad" (MO_US 2).

"Es como un efecto de pedantería, o de poca humildad el reconocer que en realidad me gusta mandar, me gusta la gestión" (IAB_UdL 2).

Tal vez, la negación del interés por ejercer la dirección pueda atribuirse a que se percibe como una traición al espíritu colegial propio de la tradición académica, o como una banalización de la tarea en tanto la gestión ha sido tradicionalmente la menos valorada entre las funciones del profesorado universitario. Elegir esta tarea podría representar una renuncia al "núcleo" de la labor académica y suponer una "falta" de parte de quien declara su interés por la dirección.

Cabría preguntarse si este desequilibrio en el prestigio de cada aspecto de la labor académica será afectado por el incremento en la valoración de la gestión en los baremos empleados para juzgar los méritos académicos, con miras a la concesión de reconocimientos y acreditaciones. Es probable que, perteneciendo los entrevistados a una generación académica en la que la aplicación de estos mecanismos selectivos no ha tenido gran impacto, su percepción sobre el valor de la tarea gestora no se vea afectada por ellos.

En línea con lo anterior, la principal fuente de satisfacción que los participantes reconocen en el ejercicio de la gestión es el apoyo del grupo, su gratitud por la labor desempeñada y su reconocimiento. De hecho, la insistencia de los compañeros es una de las razones mencionadas con mayor frecuencia al explicar la continuidad en cargos electivos.

"si que hay renuncias pero bay compensación con la satisfacción. Si cumples tus objetivos, si el resultado sale bueno, me olvido de las horas y de la preocupación con el trabajo que hay detrás." (CF_US, 9-9). 
Otra circunstancia recurrente en el acceso a la labor gestora es el haberse producido en un momento particular de la historia organizativa de la institución, frecuentemente al inicio de un cambio. Nuevamente encontramos coincidencias con el ya mencionado estudio (Sánchez-Moreno, 2009; LópezYáñez \& Sánchez-Moreno, 2009), que mostraba que las mujeres veían facilitada su inserción en la dirección universitaria cuando la dificultad de la situación volvía poco apetecible esta tarea y/o requería una modificación en el estilo de la gestión.

"lo encontré que era muy interesante en el momento que se tenía que dar, que era en toda la transformación de los planes de estudios con la implicación de Bolonia y yo era consciente de que podía participar y de alguna forma hacer, introducir, ciertos cambios en la Facultad' (JR_UdL, 2-2).

La introducción de cambios como los supuestos por el proceso de Bolonia aparece como marco idóneo para la implicación en tareas que responden a estas demandas añadidas. Implican, además, la oportunidad de que las acciones tengan efectos fácilmente identificables, lo cual puede en sí ser fuente de satisfacción y de motivación. Es recurrente en los testimonios que se reconozca un alto grado de satisfacción por la tarea, en directa vinculación con el reconocimiento del impacto positivo del ejercicio de su función en la dinámica organizativa.

"Muy alta [la satisfacción] yo creo que todo el mundo debería pasar no tanto tiempo pero si un tiempo de su vida por puestos de gestión yo creo que te da una visión de cómo funciona la institución el mundo mucho más alla de la actividad diaria docente investigadora". (JP_ US, 7- 7).

\subsection{En relación con las preocupaciones en el ejercicio del cargo}

Dada la relevancia que el interés y el beneficio colectivo adquieren en la motivación para asumir la gestión y en la satisfacción por su ejercicio, no es extraño que una de las principales preocupaciones sea la de no cumplir estos objetivos. En este sentido, el surgimiento de conflictos interpersonales en la organización representa un motivo de desasosiego y gestionarlos aparece como una de las demandas de la tarea que más esfuerzo requiere y donde mayores necesidades de formación se reconocen.

Coincidiendo con Castro (2010), aquellos factores que tienden a limitar la autonomía en las decisiones y el ejercicio de la función son causas de preocupación o dificultad. Entre ellos, se señalan el tipo de relación con los órganos colegiados o la existencia de grupos de presión. El manejo de las posibles injerencias de unos y otros representa un desafío complicado de solventar. 
Asimismo, habiendo sido la tarea de gestión tradicionalmente infravalorada, no es sorprendente que una de las preocupaciones principales que genera su ejercicio esté relacionada con el perjuicio que ocasiona en la investigación. Así lo subraya una de las participantes en la segunda ronda de entrevistas:

"Si te metes en gestión y lo otro lo dejas, te desenganchas..." (MO_US 2).

Aunque resulta inevitable que las labores de docencia y de investigación sean afectadas por el ejercicio de la gestión, los gestores van aprendiendo estrategias para superar esta dificultad como delegar determinadas tareas, a fin de liberar las propias energías para realizar las que suponen mayor responsabilidad.

"Y lo menos gratificante o más difícil de la gestión es saber delegar. Creo que es con lo que tenemos más dificultad porque tenemos la idea de que gestionar es controlar todo. En parte, es así, pero no supone la ejecución de todo." (JM_US, 19-19).

Parece que el paso de la etapa inicial a la de desarrollo, en el "ciclo vital" (Castro, 2010), está determinado por la adquisición de capacidades que disminuyen el potencial lesivo del ejercicio del cargo sobre el conjunto de la identidad académica.

En buena medida, dicha capacidad de delegar es posibilitada por la adquisición de una visión más global de la institución universitaria, que se reconoce como un conocimiento valioso adquirido en la gestión. Tan importante se ve alcanzar esta perspectiva general que muchos participantes consideran recomendable que todos los académicos ocupen un cargo de gestión a fin de alcanzar este saber.

"Yo considero que la gestión debería ser obligatoria, que cada diez años de vida académica debería haber un mínimo de dos de gestión [...]-Creo que, a ciertos niveles, no es compatible con la docencia porque se hace craso favor los estudiantes". EP_US, 8- 8 .

"Todo el mundo debería pasar [por la gestion] en algún momento, pero sobre todo porque cuando uno ha pasado por un cargo de estos ve las cosas de otra forma, es decir antes de pasar por un cargo [...] ve [...] su grupo de investigación como el núcleo de todo pero cuando uno pasa a un cargo [...] ves que los demás también tienen ombligo. Y esto es importante" TG_UdL, 12-12.

Estas ideas remarcan nuevamente la relevancia de los aspectos social e interpersonal en la labor gestora que, como mostraremos, se refleja en las necesidades formativas identificadas. 


\subsection{En relación con la necesidad de formación previa en gestión}

En contraste con la comentada ausencia de formación específica se admite la conveniencia de contar con conocimientos sobre gestión universitaria al asumirla. Son muy pocos quienes rechazan esta idea y se apoyan para hacerlo en el temor a que tal preparación suponga una especialización que aleje al gestor de la labor académica.

La preocupación por las relaciones sociales aparece como una de las competencias que se admiten como necesarias en la dirección universitaria, en contraste con las tareas de tipo estratégico priorizadas por estudios como el de Maassen y Pausits (2013). No existen diferencias significativas entre los participantes, que pudieran ponerse en relación con el ámbito o nivel en que ejercen la gestión o con el género. Sólo se perciben ligeras diferencias en el modo en que estas capacidades son enunciadas o ejemplificadas, que pueden derivarse de la imagen con que cada gestor las vincula-más o menos cercana a su experiencia directa- y al grado de conocimiento teórico sobre el tema, que condiciona el lenguaje empleado.

El conocimiento de la normativa y las capacidades para el manejo económico también se señalan como saberes pertinentes aunque con menor énfasis, tal vez porque el apoyo del personal de administración puede suplir la escasez de conocimiento del gestor académico.

Particularmente valoradas por nuestros informantes son las habilidades sociales. Pese a que la relación entre éstas y la propia personalidad de los sujetos crea dudas en algunos, hay amplio acuerdo en la conveniencia de su aprendizaje. Asimismo, las habilidades mencionadas por los participantes en nuestro estudio coinciden con las citadas en la literatura:

- la capacidad de liderazgo y dirección estratégica, orientada a motivar al grupo y movilizar esfuerzos en pro de las ideas defendidas (Kezar \& Lester, 2009; Bryman, 2007; Blackmore \& Blackwell, 2006; Scott \& Webber, 2008).

- la empatía y las capacidades de escucha y de apoyo, así como de construcción de sentimientos de grupo (Kezar \& Lester, 2009; Bryman, 2007; Scott \& Webber, 2008, Spendlove, 2007; Wolverton et al., 2005).

- la de transmitir confianza, credibilidad y disponibilidad (Kezar \& Lester, 2009; Bryman, 2007).

"Competencias para ser un gestor: persona con habilidades personales para liderar grupos, sin afán de protagonismo, capaz, de hacer creer al gente el espiritu de grupo y de co-responsabilizar tomas de decisiones en el sentido que este grupo que ha estado trabajando vea la decisión que has tomado (...) que ellos tuvieron alli su granito de arena, el hacer participar, recoger bien las necesidades de la gente". (CM_US, 14- 14). 
"..., he aprendido que hay que tener mucha mano izquierda, que no hay que fomentar las diferencias sino lo contrario. Saber contemporizar, quitarle bierro a las cosas y hacer, a veces, de mediador si esas personas están dispuestas. Es difícil. Hay que tener habilidades y cierto carácter de tranquilidad y de mesura para poder afrontar eso y tener buenas relaciones con el PAS. Eso es clave. Saber que cada uno debe estar en su sitio, saber exigir lo que hay que exigiry tolerar lo que hay que tolerar, y confiar". (PE_US, 9- 10).

“...y saber gestionar emociones personales y de los demás". (GF_UdL, 9-11).

Los gestores consultados no consideran especialmente relevantes las habilidades políticas y de obtención de recursos, subrayadas por la literatura (Bryman, 2007; Kezar \& Lester, 2009; Scott \& Webber, 2008). En cambio, sí señalan el reconocimiento y la aceptación de las dinámicas instaladas en las unidades organizativas para la resolución de las tareas administrativas. Esto implica apertura de mente, humildad y capacidad para configurar equipos de trabajos potentes y cohesionados.

A pesar de la casi unánime aceptación de la necesidad de formación en gestión, se señalan múltiples obstáculos para su implementación. Además de la dificultad para desarrollar capacidades de orden personal, la escasez de tiempo y la complejidad de organizar una formación de esta índole son los más recurrentes. Para superarlos, la propuesta de una modalidad de formación semipresencial resulta la más convincente.

Asimismo, corroborando resultados de estudios previos (Aasen \& Stensaker, 2007; Calabrese et al., 2008; Sánchez-Moreno, 2008, 2009), se propone una formación basada en la simulación y en el análisis y resolución de situaciones y casos reales.

"Yo echo en falta formación práctica, de casos prácticos: tal situación, de tal forma. Creo que tiene que ser de dinámicas de grupo, de videos... Porque mi formación ha sido indirecta: en cursos que no eran de dirección propiamente pero sí tocaban competencias que se pueden poner en práctica al dirigir”. (JM_US, 8- 8).

Similarmente, estrategias de asesoramiento, acompañamiento o mentorización se valoran positivamente. En consonancia con estudios como el de Del Favero (2006) y el de Gurr, Drysdale y Mulford (2005), el acompañamiento por parte de alguien experimentado suele considerarse idóneo para la adquisición de los conocimientos que requiere la gestión universitaria. Se trataría de facilitar la incorporación de nuevos miembros y de servir de puente en las transiciones a la vez que se garantiza la continuidad del conocimiento organizativo y de las políticas de gestión. En este sentido, se considera potencialmente útil la aportación de personas expertas (Sánchez-Moreno, Murillo \& Lavié, 2010), que pudieran constituir una bolsa de asesores, es decir, de personas que hubieran ejercido la gestión y estuvieran dispuestas a prestar asesoramiento sobre ella. 
“... acompañamiento, un teléfono rojo, es decir un servicio de gente que ha pasado por cargos de gestión y te puede ayudar a pensar un problema. Un servicio disponible para efectuar consultas cuando éstas surjan" (CMG_US 2).

Casi todos los entrevistados se muestran dispuestos a participar en la formación de futuros gestores. No obstante, prácticamente ninguno ha ejercido formalmente labores de este tipo, lo que parece hallarse en relación con la escasez de mecanismos de coordinación propia de una organización débilmente acoplada, como la Universidad. Por tanto, la formación de los gestores universitarios puede ser favorable no sólo para mejorar el ejercicio de esta función sino para la del conjunto del funcionamiento organizativo, en tanto mecanismo de articulación entre sus componentes.

\section{Conclusiones}

Formulamos algunas interpretaciones y conclusiones relevantes por sus implicaciones prácticas para la mejora de la gestión académica en la Universidad.

En primer término, coincidiendo con hallazgos previos (Castro, 2010, 2011), cabe delinear cierto "ciclo vital" en el recorrido de los gestores académicos universitarios, marcado por sucesivas "fases". El acceso a la gestión aparece condicionado por cierto "compromiso personal" con alguna persona importante en la vida académica o con algún colectivo al que se debe lealtad. Este período se ve marcado por el sentimiento de "ponerse a prueba", enfrentando situaciones desafiantes por su complejidad.

Tras la superación de esa "prueba iniciática", en una segunda fase se realiza un decidido ejercicio del cargo alentado por los logros obtenidos y por la satisfacción vinculada al reconocimiento del impacto positivo del ejercicio de su función en la dinámica de la organización. Coincidentemente, el sentimiento de eficacia parece ser el factor más influyente en el deseo de ejercer la gestión e, incluso, en la aspiración de hacerlo en niveles de mayor responsabilidad (Kwan, 2009).

Finalmente, se observan dos tendencias. Una de desapego y preparación para la retirada y una reiterada voluntad de permanecer en la gestión sea repitiendo el mismo cargo o asumiendo otro. Esta permanencia se funda en la convicción de no poder declinar la responsabilidad sobre un proyecto valioso de cuya continuidad se es, en gran parte, responsable.

Una mirada al ciclo descrito evidencia la contradicción entre la ausencia de referencias al interés "personal" como motivación para acceder y mantenerse en cargos de gestión y la tendencia a continuar en su ejercicio. Cabe preguntarse si se vincula con cierto sentimiento de vergüenza al reconocer el gusto 
por dirigir, que podría relacionarse con, al menos, dos cuestiones relativas a la identidad profesional académica. En primer lugar, el carácter horizontal y democrático que puede reclamarse para las relaciones entre miembros de la comunidad científica; por otra parte, la gestión ha tendido a verse como la función menos prestigiada entre las propias del profesorado universitario. Aceptar el gusto por ella podría suponer el reconocimiento de disfrutar de una tarea poco valorada.

En cualquier caso, es inevitable preguntarse qué razones subyacen a la citada tendencia a continuar en la gestión académica. Una posible respuesta surge de la consideración de un cierto miedo de la vuelta a la nada, entendido como la desposesión de la capacidad formal para influir en los demás. Otra posible explicación puede ser el temor a las posibles represalias de compañeros que se hubieran sentido perjudicados por decisiones adoptadas durante el ejercicio del cargo. Este temor se relaciona con el sentimiento de valoración derivado de los logros obtenidos durante el ejercicio de los cargos de gestión, al que el abandono de la gestión supondría renunciar. Con respecto a esto último, la insistencia en no haber recibido formación $-\mathrm{y}$, en muchos casos, de no haberla necesitado- en contraste con el reconocimiento de su virtual utilidad podría interpretarse como un indicador de la capacidad innata del académico para la labor gestora.

En este sentido, la preocupación por los conflictos interpersonales podría explicarse porque su aparición genera dudas sobre el apoyo de los compañeros, que es la fuente de legitimidad del gestor.

Como contrapartida de esta preocupación por el nivel interno o "micro", resulta atendible la escasa alusión a cuestiones de orden general o "macro", tales como el impacto de cambios sociales y las presiones del mercado que generan -según el estudio de Savage (2013: pp. 100) "angustia, frustración y miedo" en muchos líderes educativos. Cabe preguntar si esto se debe a que no afecta directamente al entrevistado o, por el contrario, porque se considera ajeno a las propias posibilidades de actuación. En cualquier caso, es indudable que este tipo de debates, que conduciría a poner en discusión el modelo de universidad implicaría atender a la dimensión de "la política". Cabría pensar que, al ver estas cuestiones como ajenas a su ámbito de intervención directa, considerarlas se convertiría en una limitación del propio poder. Por el contrario, sus efectos -fundamentalmente, los negativos- en el interior de las organizaciones refuerzan la necesidad de poner en juego las valoradas habilidades sociales a fin de contrarrestarlos. En este sentido, supondrían un mecanismo indirecto de fortalecimiento del poder de las figuras gestoras.

La formación para la gestión es considerada útil siempre que se ajuste a algunos principios coincidentes con los indicados por la bibliografía. Destacan entre ellos el carácter práctico que dé lugar al aprendizaje por la experiencia (Aasen \& Stensaker, 2007; Calabrese et al., 2008), la observación y el modelado, 
contemplando estrategias de tutorización, mentorización y la inclusión de la formación como estrategia que dé continuidad a la gestión (Mc Murray et al., 2012).

En relación con los contenidos, aunque se reconoce la relevancia de conocimientos relativos al ámbito formal (legislación, conocimientos informáticos, planificación estratégica...), tienen prioridad las habilidades sociales -en particular, para la resolución de conflictos y el manejo de grupos.

\section{Implicaciones}

Tal como se señalara previamente, una de las finalidades del estudio cuyos resultados venimos comentando era el diseño de un Programa Formativo dirigido a Académicos Gestores Universitarios -cuya primera edición ya ha tenido lugar- que contemplara las aportaciones reseñadas. Particular atención recibió el análisis de los aspectos sociales del funcionamiento organizativo, a los que se dedicaron tres de los cinco módulos en que se organizó el Programa. Cuestiones como las habilidades sociales necesarias para dinamizar los grupos de trabajo y las capacidades de gestión de las propias emociones fueron especialmente contempladas, así como otras relacionadas con la dimensión informal de las organizaciones, tales como el poder, el liderazgo y la cultura organizativa. Creemos que, en consonancia con los hallazgos aquí presentados, la atención a estos elementos es central en la formación de los gestores académicos universitarios. No sólo suponen una aportación que puede incidir en la mejora del funcionamiento de las instituciones universitarias, sino también una contribución al bienestar de quienes ejercen una labor estresante como es la gestión de organizaciones complejas.

Asimismo, a partir de los resultados obtenidos en esta investigación, se procuró la adopción de una metodología de índole práctico, que permitiese recuperar el conocimiento de los participantes como insumo para el debate y la reflexión colectivos. A la luz de los conceptos aportados por el Programa, estas experiencias - propias o de carácter vicario y derivadas de las situaciones dilemáticas propuestas como objeto de discusión- se convierten en punto de partida de aprendizajes situados y relevantes, debido a su clara vinculación con la realidad universitaria. 


\section{Limitaciones y líneas futuras de investigación}

Pese a los valores que desde nuestro punto de vista tiene el estudio presentado, algunas limitaciones deben señalarse. Aunque entre ellas cabe mencionar el tamaño relativamente pequeño de la muestra, entendemos que ésta ha de ser relativizada dado que se trata de un estudio de carácter exploratorio. En ese sentido, sería oportuna e interesante la realización de una indagación más amplia con finalidad confirmatoria.

Por otra parte, entendemos que nos encontramos en un momento de transición en el modelo de gobierno y gestión de las universidades españolas. Como consecuencia, los resultados obtenidos pueden estar reflejando una realidad que en breve no se corresponda con la predominante. Sin embargo, en cualquiera de los posibles modelos de gestión existentes, la formación ha de ocupar un lugar destacado y en ese sentido los hallazgos presentados pueden resultar interesantes y pertinentes. Investigaciones futuras sobre la gestión universitaria y la formación para su ejercicio podrían focalizarse sobre los cambios en las demandas que esta tarea plantea a los responsables de ejecutarlas.

En otro orden de cosas, sería pertinente estudiar el impacto de una formación de las características de la aquí propuesta tanto en el funcionamiento de las organizaciones universitarias como en la autopercepción de sus gestores respecto de su aportación a las instituciones. En definitiva, se trata de un campo de estudio amplio y rico que, pese a su relevancia, aún resulta poco explorado.

\section{Referencias}

Aasen, P., \& Stensaker, B. (2007). Balancing trust and technocracy? Leadership training in higher education. International Journal of Educational management, 21(5), 371-383. http://dx.doi.org/10.1108/09513540710760165

Blackmore, P, \& Blackwell, R. (2006). Strategic leadership in academic development. Studies in Higher Education, 31(3), 373-387. http://dx.doi.org/10.1080/03075070600680893

Bryman, A. (2007). Effective leadership in higher education: A literature review. Studies in Higher Education, 32(6), 693-710. http://dx.doi.org/10.1080/03075070701685114

Calabrese, R., Roberts, B.B., McLeod, S., Niles, R., Christopherson, K., Singh, P et al. (2008). Emerging technologies in global communication. Using appreciative inquiry to improve the preparation of school administrators. International Journal of Educational management, 22(7), 696-709. http://dx.doi.org/10.1108/09513540810908593 
Castro, D. (2010). El desempeño de la gestión en la Universidad: El caso de Decanos y Directores de Departamentos. Educación XXI, 13(2), 217-239.

Castro, D. (2011). La gestión universitaria. Una aproximación a la dirección de centros y departamentos. Zamora: Comunicación Social.

Corominas Subias, A., \& Sacristán Adinolfi, V. (2011). Las encrucijadas estratégicas de la universidad pública española. Revista de Educación, 355, 57-81.

Deem, R. (1998). 'New managerialism' and higher education: The management of performances and cultures in universities in the United Kingdom. International Studies in Sociology of Education, 8(1), 47-70. http://dx.doi.org/10.1080/0962021980020014

Deem, R. (2005). Management as ideology: The case of 'new managerialism' in higher education. Oxford Review of Education, 31(2), 217-235. http://dx.doi.org/10.1080/03054980500117827

Deer, C. (2003). Bourdieu on Higher Education: the meaning of the growing integration of educational systems and self-reflective practice. British Journal of Sociology of Education, 24(2), 194-207. http://dx.doi.org/10.1080/01425690301904

Del Favero, M. (2006). Disciplinary variation in preparation for academic dean role. Higher Education Research and Development, 25(3), 277-292. http://dx.doi.org/10.1080/07294360600793069

Goodwill, R. (2012). Engaging staffa communities in a knowledge transfer strategy: A case of study at the University of Melbourne. Journal of Higher Education Policy and Management, 34(3), 285-294. http://dx.doi.org/10.1080/1360080X.2012.678726

Gurr, D., Drysdale, L., \& Mulford, B. (2005). Successful principal leadership: Australian case studies. Journal of Educational Administration, 43(6), 539-551. http://dx.doi.org/10.1108/09578230510625647

Jiménez-Contreras, E., De Moya Anegón, F., \& Delgado López-Cózar, E. (2003). The evolution of research activity in Spain. The impact of the National Commission for the Evaluation of Research Activity (CNEAI). Research Policy, 32, 123-142. http://dx.doi.org/10.1016/S0048-7333(02)00008-2

Kezar, A., \& Lester, J. (2009). Supporting Faculty Grassroots Leadership. Research on Higher Education, 50, 715-740. http://dx.doi.org/10.1007/s11162-009-9139-6

Kwan, P. (2009). Vice-principals' dilemma-career advancement or harmonious working relationship. International Journal of Educational Management, 23(3), 203-216. http://dx.doi.org/10.1108/09513540910941711

Leadership Foundation for Higher Education (2009). Disponible online en: http://www.lfhe.ac.uk/en/governance-new/index.cfm 
López-Yáñez, J., \& Sánchez-Moreno, M. (2008). Women leaders as agents of change in higher education organizations. Gender in Management: An International Journal, 23(2), 86-102. EEUU: Emerald Group Publishing Limited. http://dx.doi.org/10.1108/17542410810858303

López-Yáñez, J., \& Sánchez-Moreno, M. (2009). Mujeres agentes de cambio en la dirección de organizaciones universitarias. Revista de Educación, 348, 331-353.

Maassen, P., \& Pausits, A. (2013). Higher education management programmes in Europe: From grassroots to sustainable developments and impact. En B.M. Kehm, \& C. Musselin (Eds.), The Development of Higher Education Research in Europe (pp. 69-86). Rotterdam: Sense Publishers.

Mc Murray; A.M., Hnely, D., Chaboyer, W., Clapto, J., Lizzio, A., \& Teml, M. (2012). Leadership succession management in a university health faculty. Journal of Higher Education Policy and management, 34(4), 3365-3376.

Murillo García, F., \& Salas Durazo, I.A. (2011). Gestión universitaria y estilos de liderazgo. Cuadernos de Educación y Desarrollo, 3(30). Disponible online en: http://www.eumed.net/rev/ced/index.htm

Nguyen, T.L.H. (2012). Identifying the needs of Heads of Department in a newly establishhed universiy in Vietnam. Journal of Higher Education Policy and Management, 34(3), 309-321. http://dx.doi.org/10.1080/1360080X.2012.678730

Pulido, J., Aguilar, V., \& Alemán, J. (2010). La formación de directivos universitarios en España, Reino Unido, Francia y Alemania. En A. Manzanares (Ed.), Organizar y dirigir en la complejidad. Instituciones educativas en evolución (pp.572-585). Madrid: Wolters Kluwer.

Sánchez-Moreno, M. (2008). Mujeres directivas: Un estudio en la Universidad española. Sevilla: Secretariado de Publicaciones de la Universidad.

Sánchez-Moreno, M. (ed.) (2009). Mujeres dirigentes en la Universidad. Las texturas del liderazgo. Zaragoza: Prensas Universitarias de Zaragoza.

Sánchez-Moreno, M., \& López-Yáñez, J. (2013). Buenas prácticas de gobierno y gestión en la Universidad. Teoría de la Educación, 25 (1), 125-148.

Sánchez-Moreno, M., Murillo, P., \& Lavié, J.M. (2010). La investigación de la gestión universitaria: conociendo buenas prácticas en Manzanares. En A. (Ed), Organizar y dirigir en la complejidad. Instituciones educativas en evolución (pp.486-499). Madrid: Wolters Kluwer.

Savage, G. (2013). Governmentality in practice: Governing the self and others in marketized education system. En D. Gillies, Educational Leadership and Michel Foucault. London: Routledge. 
Scott, S., \& Webber, C. (2008). Evidence- based Leadership Development: The 4L framework. Journal of Educational Administration, 46(6), 762-776. http://dx.doi.org/10.1108/09578230810908343

Scott, G., Coates, H., \& Anderson, M. (2008). Academic Leadership Capabilities for Australian Higher Education. Australia: University of Western Sydney and Australian Council for Educational Research.

Sharrock, G.S. (2007). After Copernicus: Beyond the crisis in Australian universities. Australian Universities Review, 49, 2-14.

Solé Parellada, F., \& Llinàs-Audet , X. (2011). De la burocracia profesional a la tecnópolis: Los desafíos estratégicos de la gestión universitaria. Revista de Educación, 355, 17-30.

Spendlove, M. (2007). Competencies for effective leadership in higher education. International Journal of Educational Management, 21(5), 407-417. http://dx.doi.org/10.1108/09513540710760183

Wolverton, M., Ackerman, R., \& Holt, S. (2005). Preparing for leadership: What Academic Department Chairs Need to Know. Journal of Higher Education Policy and Management, 27(2), 227-238. http://dx.doi.org/10.1080/13600800500120126

Intangible Capital, 2016 (www.intangiblecapital.org)

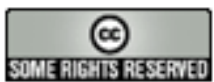

Article's contents are provided on an Attribution-Non Commercial 3.0 Creative commons license. Readers are allowed to copy, distribute and communicate article's contents, provided the author's and Intangible Capital's names are included. It must not be used for commercial purposes. To see the complete license contents, please visit http://creativecommons.org/licenses/by-nc/3.0/. 